\title{
Unexplained Recurrent Fevers and the Importance of Inquiring About Occupation: A Case Report
}

\author{
Zachary Reese, MS4 \\ Thomas Jefferson University, zachary.reese@jefferson.edu \\ Poonam Maru, DO \\ Thomas Jefferson University, poonam.maru@jefferson.edu
}

Follow this and additional works at: https://jdc.jefferson.edu/tmf

Part of the Infectious Disease Commons, and the Internal Medicine Commons

Let us know how access to this document benefits you

\section{Recommended Citation}

Reese, MS4, Zachary and Maru, DO, Poonam (2016) "Unexplained Recurrent Fevers and the Importance of Inquiring About Occupation: A Case Report," The Medicine Forum: Vol. 17 , Article 11.

DOI: https://doi.org/10.29046/TMF.017.1.012

Available at: https://jdc.jefferson.edu/tmf/vol17/iss1/11

This Article is brought to you for free and open access by the Jefferson Digital Commons. The Jefferson Digital Commons is a service of Thomas Jefferson University's Center for Teaching and Learning (CTL). The Commons is a showcase for Jefferson books and journals, peer-reviewed scholarly publications, unique historical collections from the University archives, and teaching tools. The Jefferson Digital Commons allows researchers and interested readers anywhere in the world to learn about and keep up to date with Jefferson scholarship. This article has been accepted for inclusion in The Medicine Forum by an authorized administrator of the Jefferson Digital Commons. For more information, please contact: JeffersonDigitalCommons@jefferson.edu. 


\title{
Unexplained Recurrent Fevers and the Importance of Inquiring About Occupation: A Case Report
}

\author{
Zachary Reese, MS4, and Poonam Maru, DO
}

\section{INTRODUCTION}

Many different tick-borne diseases are identified in various geographical areas throughout the United States. Ixodes scapularis tick-borne diseases can present unique diagnostic challenges because of their constellation of vague symptoms. The Ixodes scapularis tick, more commonly known as the blacklegged tick, is responsible for three specific tick-borne infections: Lyme disease (Borellia burgdorferii), Babesiosis (Babesia microti), and Anaplasmosis (Anaplasma phagocytophilum) also known as human granulocytic anaplasmosis. Although these three tick-borne diseases are characteristically found in the New England area, the geographical distribution of the Ixodes tick has expanded to encompass much of the northeastern United States and even states in the upper mid-west. ${ }^{1}$ Although Lyme disease is the most prevalent of the tick borne illnesses, the incidence of Babesiosis has increased exponentially in the last 50 years and cases have recently been reported in 22 states. ${ }^{2}$ Here we present a case of Babesiosis which was diagnosed only after an occupational history detailing tick exposure was obtained.

\section{CASE PRESENTATION}

A 53-year-old Caucasian male with no significant past medical history presented to an emergency department in Delaware during late June with a three-day history of persistent fevers, two episodes of syncope upon standing, drenching sweats, and dark urine. The previous day, he had been evaluated at another hospital with similar symptoms; there, a CT scan of his abdomen showed non-obstructing kidney stones and was negative for any renal masses, bladder masses, or urinary obstruction. He was diagnosed with nephrolithiasis, treated with intravenous fluids, and discharged. Due to persistence of the dark urine, fevers, and drenching sweats, he presented to Thomas Jefferson University Hospital emergency department. A further history obtained revealed an unremarkable past medical and past surgical history, and he denied taking any medications. The patient admitted to "significant" tobacco use, some marijuana use, and occasional cocaine use.

On presentation, the patient was febrile with temperature $104.5^{\circ} \mathrm{F}$, and tachycardic with heart rate of 102 beats per minute. He was normotensive, his respiratory rate and oxygen saturation on room air were within normal limits. On physical exam, the patient was diffusely diaphoretic and tachycardic. Pertinent laboratory testing revealed a normal basic metabolic panel, and normal liver function tests. A complete blood count showed a normal hemoglobin, normal white blood cell count with a differential of $10 \%$ bands and $12 \%$ monocytes, and a platelet count of $65,000 / \mathrm{mcL}$. Urinalysis showed a large amount of gross blood in the urine, urobilinogen, and granular casts. The patient was admitted for further workup.

The cardiac workup that was performed to evaluate the patient's complaint of syncope, including electrocardiogram, echocardiogram, and stress test, were unremarkable. Upon further questioning, it was discovered that the patient performed landscaping work and spent much time trimming trees.

\section{DIFFERENTIAL DIAGNOSIS}

The differential diagnosis for this patient with recurrent fevers, thrombocytopenia, macroscopic hematuria, and recurrent syncope was rather broad. A more common systemic bacterial infection was initially suspected due to the patient's persistent fevers, but this was ruled out when routine urinalysis came back negative for markers of infection, and when blood cultures did not grow any bacteria. The persistent hematuria in this patient with significant smoking history was concerning for a bladder malignancy, which was ruled out with a CT scan. Recurrent syncope indicated a possible cardiac etiology, including ischemic heart disease or an arrhythmia. A cardiac etiology was ruled out by the patient's negative cardiac workup. The syncope was attributed to orthostatic hypotension. This was explained by the fact that the patient had granular casts on the urinalysis, strongly suggesting that the patient had acute tubular necrosis secondary to severe dehydration, and became hypotensive upon standing. The history of recurrent fevers, coupled with the patient's occupation, was most concerning for Lyme disease, Ehrlichiosis, Anaplasmosis, or Babesiosis.

\section{OUTCOME AND FOLLOW-UP}

While in the hospital, the patient was given IV crystalloid fluid for volume resuscitation, and he was started on empiric doxycycline at a dose of $100 \mathrm{mg}$ twice daily due to concern for tick borne illness. A blood smear was obtained, and the results revealed intraerythrocytic ring forms resembling Maltese crosses, consistent with a diagnosis of Babesiosis. His antibiotic regimen was then changed to azithromycin $250 \mathrm{mg}$ daily and atovaquone $750 \mathrm{mg}$ twice daily for a total seven-day course. His symptoms resolved within 48 hours of starting the antibiotics. 


\section{DISCUSSION}

This case of a landscaper who presents at an east-coast hospital in mid-summer with recurrent fevers and thrombocytopenia fits the typical presentation of a tick-borne illness. Tick-borne illnesses carried by the Ixodes tick occur most often in the summer months with a typical prodrome comprised of fever, headache, chills, and muscle aches. With Lyme disease in particular, an erythema migrans rash may be present. These non-specific "flu-like" symptoms usually start one to four weeks after the initial infection and may be misleading if a history of tick exposure is not obtained.

Babesiosis is most commonly transmitted by the nymphal stage Ixodes scapularis tick. It is common that those infected with Babesia do not recall a tick bite because the tick, in the nymphal stage, is very small - approximately the size of a poppy seed - and can easily be missed. These ticks are most abundant in grassy or wooded areas in the summer months, most commonly June and July. The Ixodes tick is most commonly found in the Northeastern and Midwestern areas of the United States, particularly in New England, New Jersey, New York, Minnesota, and Wisconsin. ${ }^{4}$

Babesiosis is transmitted when ticks feed on the vertebrate host, effectively transmitting Babesia sporozoites to the host. These sporozoites attach to erythrocytes inside of the host, and then replicate inside of the erythrocyte. Once mature, the Babesia-infected erythrocyte bursts, and the sporozoites move to infect other erythrocytes. Because of this pathogenesis, a hemolytic picture is seen in infected patients with anemia, low haptoglobin and high lactate dehydrogenase. ${ }^{3}$

In its mildest forms, Babesiosis causes an asymptomatic infection, while moderate disease can cause fever, chills, fatigue, nausea, vomiting, and weight loss. Severe disease, which occurs in immunosuppressed patients, has been associated with adult respiratory distress syndrome, disseminated intravascular coagulation, congestive heart failure, or renal failure. ${ }^{3}$

Babesiosis is diagnosed by using a Giemsa- or Wrightstained blood smear to visualize intraerythrocytic ring forms resembling Maltese crosses. ${ }^{5}$ Alternatively, a positive Babesia polymerase chain reaction can make the diagnosis, and most patients have a relief of symptoms after a 7-day course of appropriate therapy. After treatment is initiated, symptoms usually resolve in 1-2 weeks, but anemia and thrombocytopenia can persist for several months.

It is important to note that co-infection with multiple tick-borne diseases is also common. Nearly one-third of patients from endemic areas who are infected with Babesiosis are co-infected with Lyme disease. Further, while erythema migrans is present in $90 \%$ of people that are infected with Lyme disease alone, only $80 \%$ of patients co-infected with Lyme disease and Babesiosis have evidence of erythema migrans. ${ }^{5}$ Because co-infection is common, if a tick-borne illness is suspected, a complete blood count and a blood smear should be obtained. If a tick-borne infection is diagnosed, it is important to test for other Ixodes tick-borne diseases as well. This case also highlights the importance of detailed history taking, especially regarding occupation and recent outdoor activity. Obtaining an extensive history can help to keep tick-borne diseases on the list of differential diagnoses.

\section{KEY POINTS}

- Remember to include tick-borne diseases on the differential with a patient who presents with recurrent fevers and thrombocytopenia in the summer months.

- Consider gathering an extensive and thorough social history in patients who present with a vague constellation of symptoms.

\section{REFERENCES}

1. Nathavitharana, R.; Mitty, J. Diseases from North America: Focus on Tick-Borne Infections. CME Tropical Medicine. 2015; 15: 74-77.

2. Vannier, E.; Diuk-Wasser, M.; Mamoun, C.; Krause, P. Babesiosis. Infect Dis Clin NAm. 2015; 29: 357-370.

3. Vannier, E.; et. al. Human Babesiosis. N Engl J Med. 2012; 366(25): 2397-2407.

4. Parasites - Babesiosis. http://www.cdc.gov/parasites/babesiosis. 4 February 2014

5. Gutman, J.; et. al. A 60-Year-Old Man with Fever, Rigors, and Sweats. N Engl J Med. 2003; 349: 1168-1175. 